\title{
Naturalism and Non-Qualitative Properties
}

Sam Cowling, Denison University

[Penultimate draft; please cite published version]

Forthcoming in Common Sense Metaphysics: Themes from the Philosophy of Lynne Rudder Baker (eds.

Luis Oliveira and Kevin Corcoran)

Lynne Baker's case for the incompatibility of naturalism with the first-person perspective raises a range of questions about the relationship between naturalism and the various properties involved in first-person perspectives. After arguing that non-qualitative properties - most notably, haecceities like being Lynne Baker - are ineliminably tied to firstperson perspectives, this paper considers whether naturalism and non-qualitative properties are, in fact, compatible. In doing so, the discussion focus on Shamik Dasupgta's argument against individuals and, in turn, non-qualitative properties. Several strategies for undermining Dasgupta's argument are considered, drawing on de re laws and haecceitistic possibilities. Finally, an analogy is drawn between naturalism and platonism regarding mathematical entities and naturalism's parallel commitment to individuals. I conclude that naturalists are obliged to posit non-qualitative properties.

\section{§1. Introduction}

Lynne Baker believed in the importance of metaphysics and the significance of its central questions. While some of us are perfectly content to bat around debates regarding tropes and universals or litigate the abstract-concrete distinction, Baker always kept her focus trained on abiding metaphysical concerns, especially those regarding our place in the world. Consider the foundational issue she sets out at the beginning of Naturalism and the First-Person Perspective:

[The robust first-person perspective] can neither be eliminated nor reduced to anything nonperspectival or non-first-personal. It is, in fact, a dispositional property that must appear in basic ontology. If that is correct, then there are genuinely first-person properties. Since the scientific picture of the world is of a totally objective, totally impersonal reality, the scientific picture cannot accommodate the first-person perspective.... The first-person perspective is a serious challenge to naturalism. (2013: xxi)

This challenge, which is at the heart of Baker's final book, takes the form of what others might call a "location" or "placement" problem. ${ }^{1}$ Given the ontology and ideology of contemporary naturalistic metaphysics, where do we as bearers of first-person perspectives fit into the world? According to Baker, naturalists are obliged to admit that the answer is "nowhere," since neither the ontology nor ideology of naturalism can aptly characterize first-person perspectives. And, since the existence of our first-person perspectives isn't an open metaphysical question, the inability of naturalists to place us in their picture of reality is reason to deny that contemporary naturalism provides an exhaustive description of the world. But, since naturalism purports to give a complete picture of the world, we are required to reject naturalism. According to Baker, the

\footnotetext{
${ }^{1}$ I take the terminology of "placement problems" from Price (2011) and treat it interchangeably with talk of "location problems," drawn from Jackson (1998), though I assume neither Price's nor Jackson's views of what it would mean to satisfactorily address such problems.
} 
kinds of things we are-namely, persons-simply can't be reduced to anything within the naturalist's impersonal world. And so the naturalist's ambition of supplying us with the full metaphysical story cannot be fulfilled.

When understood as a placement problem, Baker's challenge to naturalism is of clear importance but it is also conspicuously multi-faceted. Notice that the first-person perspective is a complex phenomenon. According to Baker, a rudimentary first-person perspective requires both sentience, understood as a "primitive form of consciousness" (41), and intentionality, which requires "minimal agency [and] goal-directed behavior." (41) So, when it comes to the challenge of placing rudimentary first-person perspectives in the world, would-be naturalists run headfirst into two narrower placement problems: one regarding the phenomenal properties that attend sentience and another regarding semantic properties, which are bound up with intentionality and mental content. ${ }^{2}$ Once we turn to what Baker calls the robust first-person perspective, placement problems continue to multiply, since a robust first-person perspective requires the potential for self-reflection as well as a capacity to think about one's self in first-personal terms. ${ }^{3}$ The manifestation of indexical thought and other sophisticated cognition therefore yield yet more placement problems (or more complex variants of pre-existing ones), each of which are downstream from those more basic ones concerning phenomenal and semantic properties. ${ }^{4}$

There is, of course, a wealth of philosophical work focused on placement problems regarding phenomenal and semantic properties. And, if it should turn out that these or any other properties constitutive of first-person perspectives cannot be reconciled with the naturalist's inventory of reality, Baker's overall judgment will be vindicated. But, while Baker's focus on the first-person perspective-as a unitary, broad phenomenon-highlights a core challenge for naturalists, I believe would-be naturalists are better served to disentangle, as best they can, these various placement problems and address them in a piecemeal fashion. In this spirit, my project in this paper is not to review the status of the familiar placement problems regarding naturalism and phenomenal or semantic properties. Instead, I will examine an additional though largely neglected placement problem that is also required for providing a metaphysics of first-person perspectives. This placement problem concerns non-qualitative properties such as haecceities like being Lynne Baker which play an ineliminable role in understanding first-person perspectives. This placement problem asks how, if at all, we can make sense of the property of being Lynne Baker in a naturalistic conception of reality, which gives pride of place to qualitative properties like mass, charge, and shape.

Why think that we can't account for first-person perspectives without positing nonqualitative properties? Because first-person perspectives are tied to specific individuals in a manner distinctive to non-qualitative properties. My first-person perspective is distinguished by virtue of being the perspective available only to the bearer of being Sam Cowling. Indeed, even if another perspective were experientially indiscernible-i.e., were precisely the same "from the inside" - it would nevertheless be distinct from my own first-person perspective. This is because

${ }^{2}$ If phenomenal properties are reducible to semantic or intentional properties or, alternatively, if semantic properties reduce to phenomenal ones, it will turn out that we have a uniquely fundamental placement problem, though we would still require an account of how the semantic fixes the phenomenal (or vice versa). ${ }^{3}$ See Baker (2013: 40-46).

${ }^{4}$ Throughout what follows, I take talk of "properties" to subsume properties as well as relations. 
each first-person perspective is modally tied to a specific individual, remaining uniquely connected to that individual even if they might have qualitatively indiscernible doppelgangers. This suggests that, while some qualitative properties might be necessary conditions for having a first-person perspective, the modal character of perspectives-specifically, their tie to identities rather than qualities - requires that they are either non-qualitative or, at a minimum, have nonqualitative properties as parts or constituents. ${ }^{5}$ Put differently: the instantiation of first-person perspectives is tethered to specific individuals in a way that transcends qualitative properties and so must be at least partly non-qualitative.

To see why Baker holds the placement of first-person perspectives to involve nonqualitative properties, consider one of her focal questions used to draw out the tension between naturalism and the first-person perspective: "How can we understand the apparent fact that I am LB?"(1) At the end of Naturalism and the First-Person Perspective, Baker sketches her answer.

First, "let $F$ be the dispositional property expressed by 'a first-person perspective.'"(180) Then, says Baker, "[w]e are now in a position to understand how my being LB is a fact. The key is that personal identity can be understood in terms of haecceities... 'I am LB' expresses a fact if and only if my haecceity is my-exemplifying-F-essentially and LB's haecceity is also myexemplifying-F."(182) Put roughly, Baker's proposal is that some non-qualitative propertiese.g., haecceities like being Lynne Baker-are identical with specific instances of a fundamental dispositional property, having a first-person perspective. In effect, Baker's proposes that we reduce non-qualitative properties like being Lynne Baker by identifying them with specific instances of the property of having a first-person perspective.

If Baker is correct, there is no way of making sense of the first-person perspective or placing it into the world without appeal to some property like being this very thing or being Lynne Baker, which on her view traces back to the particularity of specific instances of having a first-person perspective. ${ }^{6}$ So, if our ambition is to account for facts like "I am LB" in the world of the naturalist, we will, at some point, face the difficult question of what naturalists should make of nonqualitative properties and, more specifically, haecceities such as being Lynne Baker.

In what follows, I examine the nature of non-qualitative properties with an eye towards the placement problem that arises when we ask where, if at all, they fit into a naturalistic

\footnotetext{
${ }_{5}^{5}$ Some might be tempted to identify individual perspectives with haecceities. Setting aside whether perspectives are plausibly held to fall within the ontological category of property, there is good reason to reject such a view. It seems that I could exist without my perspective-e.g., the object with which I am identical might never have achieved the cognitive benchmarks required for bearing a perspective. Those like Baker, who hold that we are essentially perspectival beings, will disagree. Note, however, that such views likely require perspectives to be exceptionally complex dispositional properties, which are still instantiated over lengthy periods without experience (e.g., comatose humans). See Baker (2013: 128, 166). ${ }^{6}$ Baker's view requires taking haecceities to be identical to instances of the dispositional property of having a first-person perspective. It is unclear to me how a property, which is a general entity, might be identical with a particular entity like a specific property-instance. Additionally, it is not clear what, apart from haecceities, might account for the numerical difference between various instances of having a first-person perspective. Setting aside these concerns, non-qualitative properties are nevertheless clearly implicated in first-person perspectives on Baker's view. Note, for example, her claim that "self-concepts" are "nonqualitative" (33) in nature.
} 
metaphysics. I do so by exploring the most promising naturalist case against non-qualitative properties: Shamik Dasgupta's argument against the existence of individuals, which has, as an immediate consequence, the nonexistence of non-qualitative properties like being Lynne Baker. After considering a range of potential responses to Dasgupta's argument, I draw upon an analogy between individuals and mathematical entities to argue that no plausible version of naturalism licenses the elimination of individuals. I therefore conclude that naturalists are under no pressure to reject non-qualitative properties.

Before turning to the question of whether naturalism and the existence of non-qualitative properties are compatible, it will be useful to mark a complication at the heart of Baker's engagement with the placement problem regarding the first-person perspective. While our focus here will be in "carving off" non-qualitative properties from Baker's broader placement problem, I make no claim that phenomenal, semantic, and non-qualitative properties exhaust the phenomena that are constitutive of first-person perspectives. Indeed, I believe there is an ineliminable aspect of the placement problem concerned with indexicals and self-location. Like Baker, I remain concerned with how to account for expressions like ' $\mathrm{I}$ ' and "being me," but, unlike Baker, I suspect that, whatever such indexicals express, they are not properties (or at least not any remotely simple ones) and are therefore importantly different from non-qualitative properties like being Lynne Baker. ${ }^{7}$ Moreover, I see no reason to fault naturalism for omitting indexical or selflocating information from an inventory of the world. There is, I take it, no amount of information, naturalistic, non-naturalistic, or otherwise, that one can provide that will invariably close the epistemic gap between the first-personal and third-personal.

As Lewis (1979) points out, even with omniscient propositional knowledge of a world, we can still be left without resources sufficient for self-location. This is, I believe, a fact about the nature of propositional content and the distinctive character of self-locating concepts like "being me," not the ontological or ideological constraints of naturalism. For this reason, if we are unable to place the first-person perspective in the world, it will be a strike against naturalism only if it is because we are unable to place phenomenal, semantic, non-qualitative, or some other genuine sort of properties. In contrast, it will not constitute a threat to naturalism if our placement problem stems solely from challenges regarding self-locating information.

\section{\$2. Non-Qualitative Properties}

Understanding the placement problem regarding non-qualitative properties requires getting a grip on the distinctive nature of such properties. Like phenomenal and semantic properties, widespread philosophical disagreement puts any non-tendentious definition of non-qualitative properties out of reach, but some clear-cut examples can still supply us with an initial grasp on the nature of the non-qualitative.

Following Max Black (1952), let's begin by considering a world empty save for two iron spheres which are perfect duplicates of one another. These spheres are exactly alike in shape, size, internal composition, and with regard to all physical features. But, since there are two of them, they must differ in some respect. In the case of qualitatively indiscernible objects like our two

\footnotetext{
7 Throughout what follows, I assume an abundant rather than sparse conception of properties, leaving open
} whether some non-qualitative properties might be more or less fundamental. 
spheres, the relevant respects in which they differ are non-qualitative. Only one of the spheres instantiates the property of-let's stipulate-being Abe while the other one instantiates the property being Ben. And, since they agree in all of their qualitative properties whether intrinsic (e.g., being made of iron) or extrinsic (e.g., being five feet from an iron sphere), merely possible entities like Abe and Ben illustrate the fact that some properties like being Abe are non-qualitative.

The domain of non-qualitative properties includes haecceities like being Abe and being Ben but also less familiar non-qualitative properties like being five feet from either Abe or Obama. ${ }^{8}$ Paradigmatic non-qualitative properties like haecceities are modally tied to specific individuals and, as a consequence, their patterns of instantiation coincide with, not merely how individuals are, but which individuals exist. A haecceity like being Lynne Baker can have no instances if Baker does not exist and can have at most one instance if she does. In addition to haecceities, other nonqualitative properties exhibit a similar modal tie to individuals. Consider, for example, disjunctive haecceities such as being Joe or Louise and "impure" properties like being two offices down from Joe or Louise. The instantiations of such properties have obvious existential entailments-e.g., that Joe or Louise exist. Proposals for a reductive analysis of the distinction between qualitative and non-qualitative properties often seek to exploit these modal ties by holding them to be distinctive of non-qualitative properties, but, as I've argued elsewhere, these and other reductive analysis are unsuccessful. ${ }^{9}$ As a result, there is some reason to believe that the non-qualitative distinction resists any successful theoretical reduction. And, given the metaphysical import of the distinction for understanding various metaphysical notions, we are well served to treat it is a conceptual primitive which we can theorize from rather than attempt to theorize toward..$^{10}$ I'll $^{\prime}$ adopt this primitivist stance throughout what follows along with some auxiliary assumptions - most notably, that the qualitative/non-qualitative distinction is exclusive and exhaustive.

Taking the qualitative/non-qualitative distinction to be a primitive one does not mean that we can ignore substantive questions about which properties fall on either side of the divide. But, since our concern in what follows will be primarily with haecceities, we can leave aside whether various sorts of properties - e.g., modal and mathematical properties - are rightly viewed as nonqualitative. We can also set aside extant debates about the general ontological status of properties and therefore leave open whether properties are universals, tropes, primitive predicates, or what have you. It will, however, be useful to distinguish paradigmatic non-qualitative properties like being Lynne Baker from properties occasionally labelled as non-qualitative such as being selfidentical or being distinct from some actual individual. While properties of this latter sort do concern facts about the identity and distinctness of objects, they have no implications regarding the existence of specific individuals and some like being self-identical never differ between individuals. This leaves their categorization as non-qualitative properties fairly tendentious. Rather than digging in here, I'll simply refer to them as structural properties in what follows.

\footnotetext{
8 On the varieties of non-qualitative properties, see Cowling (2015) and Simmons (forthcoming).

${ }^{9}$ See Cowling (2015). On proposals for analyzing the qualitative/non-qualitative distinction, see Adams (1979).

${ }^{10}$ Here, I assume that we can take either qualitative or non-qualitative as a primitive notion and define the distinction in keeping with assumptions about its structure-e.g., that it is exclusive and exhaustive.
} 
Before proceeding is also worth marking an apparent difference that separates the placement problem regarding the non-qualitative from those regarding phenomenal and semantic properties. While phenomenal, semantic, and non-qualitative properties are each implicated in the metaphysics of first-person perspectives, only phenomenal and semantic properties seem highly circumscribed in their distribution. For, while phenomenal and semantic properties seem to be exclusively instantiated by perspectival beings, non-qualitative properties are standardly held to be perfectly pervasive. ${ }^{11}$ Each and every object, regardless of what it is like, instantiates a haecceity along with various complex non-qualitative properties (e.g., being five feet from Obama). Since non-qualitative properties are strewn across the world, a placement problem regarding them is not exclusively concerned with first-person perspectives. It is, instead, a general concern about how non-qualitative properties might fit into a naturalistic characterization of reality. But, despite the general character of this placement problem, its resolution is still required for any successful account of how perspectives fit into the world, given the ties between perspectives and non-qualitative properties.

\section{§3. Placing Non-Qualitative Properties}

So, where do non-qualitative properties fit within a naturalistic characterization of reality? Unsurprisingly, the answer greatly depends upon what we take to be the permissible ontological or ideological inventory of naturalism. ${ }^{12}$ Instead of surveying and evaluating every variety of naturalism (which would be all but impossible), I focus on what seems the most promising way to see whether non-qualitative properties resist placement within a naturalistic metaphysics: assess the strongest naturalistic argument against their existence-namely, an argument that wields naturalistic considerations against non-qualitative properties. If such an argument succeeds, then we have reason to think that, just as Baker claims first-person perspectives cannot fit within a naturalist metaphysics, non-qualitative properties are similarly prohibited by a

commitment to naturalism. If, however, naturalistic considerations do not provide reason for us to reject non-qualitative properties, we would have fair evidence for their compatibility with naturalism.

It is convenient, then, that the strongest case for rejecting non-qualitative properties - set out in Dasgupta (2009) - is premised upon the application of naturalistic commitments to our best

\footnotetext{
${ }^{11}$ While I will speak throughout about individuals instantiating phenomenal, semantic, and non-qualitative properties, it is highly plausible that temporary states and proper parts of individuals instantiate properties of these sorts as well. The relationship between the properties of individuals and their states or parts is, of course, a topic worthy of its own discussion. Here, I largely set aside these concerns and take the following conclusions regarding non-qualitative properties to hold, with suitable modifications, regardless of one's views about the relationship between individuals, their states, and their parts.

12 Varieties of naturalism are about as numerous as naturalists and, while Baker's claims are plausibly true of some varieties of naturalism, it is unclear how widespread they are among naturalists. Notice, for example, that Baker's discussion of naturalism also seems to immediately rule out the possibility of a view like Chalmers (1996: 113) on which naturalism and dualism are paired together.
} 
metaphysical theories. ${ }^{13}$ In order to assess Dasgupta's argument, we first need to orient ourselves in the relevant space of views regarding individuals and non-qualitative properties. (An important qualification before proceeding: Dasgupta makes no general claim to naturalism. For our purposes, the crucial point is that the argument he deploys is a naturalistic or, as Turner (2017) puts it, a "broadly scientific" one.

Following Dasgupta, let's distinguish between two kinds of fundamental facts. Facts like the one expressed by "Something is brown" are qualitative while facts like the one expressed by "Edie is brown" are individualistic. According to qualitativism, the fundamental facts about the world are exclusively and exhaustively qualitative; they do not involve or entail the existence of any specific individuals. According to individualism, some fundamental facts are individualistic, so an exhaustive characterization of reality requires specifying which individuals exist. If we assume first-order logic is an apt way of representing fundamental facts, it is natural to view individualism as requiring names or individual constants for stating certain fundamental facts. Tasked with describing an especially simple world, the individualist's fundamental facts might therefore take the following form:

\section{(World) Fa \& Fb \& Ga \& Gb}

In contrast, qualitativism denies there are any fundamental individualistic facts. As a result, fundamental reality can be exhaustively described without the use of individual constants. For the qualitativist, the fundamental facts are therefore naturally expressed via the Ramsey sentence of (World), which replaces the individual constants in (World) with corresponding existential quantifiers:

(World*) $\exists x \exists y(F x \& G x) \&(F y \& \sim G y) \& \sim(x=y)$

If individualism is true, the stock of fundamental facts must fix which individuals exist. And, given individualism, it is straightforward to see why a commitment to non-qualitative properties follows. Setting aside complexities regarding the structure of facts (and keeping in mind our neutrality about whether properties are universals, tropes, or primitive predicates), one can argue as follows. Suppose the fundamental fact that Edie is brown obtains. It follows that the fact that Edie exists obtains. If that fact obtains, then the fact that something has the property of being Edie obtains. And, if something has the property of being Edie, then it must be the case that the property being Edie exists. ${ }^{14}$ Assuming that individualism differs from qualitativism by virtue of positing non-qualitative structure in addition to qualitative structure, being Edie must therefore be a non-

\footnotetext{
${ }^{13}$ A very different argument might defend a kind of structural realism that bars qualitative as well as nonqualitative properties. I set aside the prospects for such varieties of naturalism and assume that naturalism can, in principle, permits the ascription of properties to objects. Cf. Ladyman and Ross (2007).

${ }^{14}$ Given our assumed neutrality between competing views of properties, I take it that we could translate this argument into a setting amenable to the nominalist who accepts primitive predicational ideology. On that view, the question of interest isn't ontological - namely, whether being Edie exists - but ideologicalwhether "being Edie" is part of our primitive stock of predicates.
} 
qualitative property. ${ }^{15}$ In this way, a metaphysics of individuals entails a commitment to nonqualitative properties. Or, as I'll assume in what follows, the following biconditional holds: there are non-qualitative properties if and only if there are individuals. ${ }^{16} \mathrm{~A}$ case against the existence of individuals is therefore a case against non-qualitative properties.

If qualitativism is true, either the property of being Edie does not exist or it is instead a qualitative property. These options yield two importantly different qualitativist metaphysical views. Those who claim that being Edie is, in fact, qualitative must hold that objects like Edie are individuated by their qualitative properties. ${ }^{17}$ But this seems to require that being Edie is a complex qualitative property, one reducible to a conjunction of fundamental qualitative properties. Such a view is, however, rendered implausible once we note that it entails the impossibility of qualitatively indiscernible individuals. Given the apparent possibility of cases like the Abe and Ben example presented earlier, there is no good reason to deny that individuals could be qualitatively indiscernible. There is therefore little appeal in a view according to which properties like being Edie are qualitative.

According to Dasgupta, the more attractive qualitativist option dispenses, not only with individualistic facts, but with the existence of individuals altogether. For Dasgupta, this option takes the form of generalism, which disavows the apparatus of quantification for representing fundamental facts. As Dasgupta (2017: 14) describes his (algebraic) generalist metaphysics, "there are just [qualitative] properties, stitched together by the operators. No individuals in view." On such a picture of reality, Ramsey sentences encode information-e.g., information regarding structural properties - that simply isn't reflected in the world, since there are neither objects characterizable in purely qualitative terms much less individualistic facts. (Recall that structural properties are those like being distinct from something.) For the generalist, a perspicuous representation of reality must be purely qualitative since reality is exhausted by the distribution of qualitative properties. And, by rejecting quantificational structure, the generalist contends that fundamental facts are aptly expressed via a stock of predicate functors. ${ }^{18}$

\footnotetext{
${ }^{15}$ Note that my claim here is not that the existence of individuals settles the debate over the existence of properties in favor of platonism and against nominalism. Again, we've assumed here that we can treat various accounts of properties-including nominalist views that rely upon predicational ideology-are interchangeable here.

16 The truth of this biconditional depends upon a number of assumptions. Here, I set aside a range of complications including whether other ontological categories such as event bear non-qualitative properties. If they do and one accepts a metaphysical theory that admits only events, such a theory would be one way to show that individuals are not, in fact, a necessary condition for the existence of non-qualitative properties.

17 This distinction aligns imperfectly with the distinction between haecceitism, according to which there are some maximal possibilities (or possible worlds) that differ only non-qualitatively from one another, and anti-haecceitism, which rejects any purely non-qualitative variation among maximal possibilities. In Dasgupta's terminology, maximal possibilities that differ purely non-qualitatively would differ individualistically, but anti-haecceitists might endorse individualism despite rejecting the modal commitments distinctive to haecceitism.

${ }^{18}$ Dasgupta's functorese draws heavily upon Quine (1976). On functorese and its metaphysical significance, see Burgess and Rosen (1997: 185-188) as well as Turner (2011).
} 
Viewed one way, Dasgupta's generalism requires not only that we strip away the nonqualitative information in sentences like (World) that individual constants encode but that we also dispense with the structural information regarding the identity, distinctness, and number of objects the quantificational structure in (World*) encodes. Whether or not qualitativists ought to be generalists of this kind remains an open question. But, here, our interest is in whether we have naturalistically credible reasons to join Dasgupta in believing that "the world is a purely qualitative mosaic" - one without any place for non-qualitative properties. ${ }^{19}$

Dasgupta's case for qualitativism is not premised upon any claimed a priori insights into fundamental reality. Much to the contrary, it squares with the methodological scruples of naturalism, since it proceeds by noting that individualistic facts, if admitted into our metaphysics, would be both "undetectable" and "physically redundant." According to Dasgupta, the methodology of physical theory licenses a preference for theories that eschew undetectable and physically redundant structure, so we have naturalistically credible reason to prefer a qualitativist metaphysics over an individualistic one.

The core of Dasgupta's case against individuals and, in turn, non-qualitative properties, proceeds by sketching physicists' treatment of facts regarding absolute velocity within Newtonian mechanics. ${ }^{20}$ Dasgupta claims the key feature regarding absolute velocity in Newtonian Gravitational Theory (NGT) is the following: "Given any two closed systems governed by (and only by) the laws of NGT if at an initial time they differ only in facts about the absolute velocity but are exactly the same in all other respects, including all facts about relative velocity, then they will continue to be exactly the same in all those other respects at all subsequent times."(39) This fact, in turn, explains why, in worlds where NGT holds true, facts about absolute velocity are empirically undetectable and physically redundant. This motivates dispensing with any theoretical structure-e.g., absolute velocity properties or absolute velocity facts - that would correspond to "positing" absolute velocity in NGT.

According to Dasgupta, the standard response from physicists to questions about the absolute velocity of objects in NGT is that, given its undetectability and redundancy, "there is no answer: there is no such thing as... absolute velocity." (37) Since a version of NGT that retains absolute velocity structure would be, by the lights of physicists, inferior to one that eschews such structure, we have reason to prefer a version of NGT on which there are no such facts. Dasgupta takes this case study to illustrate a principle of naturalistically credible methodology: that, given otherwise equal competing theories, we have grounds for preferring the theory which dispenses with undetectable and physically redundant structure. ${ }^{21}$

Dasgupta holds that this principle for theory choice, once applied to metaphysical structure, bears against individualism and in favor of generalism. As he puts it, "primitive individuals are empirically undetectable according to our best-confirmed physics in precisely the same sense as absolute velocity is empirically undetectable according to NGT."(42) The resulting naturalistic argument against individuals therefore stems from the observation that "mere differences in individualistic facts do not give rise to any other differences at later times"

\footnotetext{
${ }^{19}$ Dasgupta (2017: 20).

${ }^{20}$ Dasgupta uses the term "primitive individuals."

${ }^{21}$ Cf. Friedman (1983: 320-335)
} 
(41).Since "[i]t is a consequence of every physical theory that we have considered over the past 400 years that primitive individuals are danglers" where "danglers" are undetectable, explanatorily redundant posits, Dasgupta concludes that "we have reason to dispense with danglers, all else being equal."(44)

The naturalistic credentials of Dasgupta's argument are about as good as one might hope to find in serious metaphysics. It relies upon no a priori or supposedly analytic theses about the structure of reality and, in keeping with familiar scientific reasoning, it generates only defeasible reasons. Dasgupta is especially clear on this point, noting that, the case against individualism hangs on the satisfaction of a relevant ceteris paribus clause: we ought to reject individualism on the grounds that individuals are danglers provided that there are no substantial theoretical gains or losses in other respects.

In virtue of the defeasible status of the considerations Dasgupta adduces, one strategy for resisting the argument is straightforward: an epistemic conservative like Baker can simply deny that all else is, in fact, equal. For the epistemic conservative, eschewing a metaphysics of individuals might yield a more virtuous physical theory, but any putative gains in that regard will be vastly outweighed by the violence that revisionary metaphysics does to our broader ontology. In offering this response, the epistemic conservative prioritizes a general metaphysical project-the accommodation of commonsense-in order to motivate the retention of an individualistic metaphysics. In doing so, she does precisely what the naturalist cannot: she draws upon considerations external to scientific theorizing like the familiar assumption that there are individuals

Of course, not all of us are epistemic conservatives. And, for would-be metaphysical naturalists, Dasgupta's argument cannot be so easily resisted. There can be no appeal to extrascientific considerations in theory choice. Nor can we simply point towards the comforts of commonsense ontology while retaining any credible claim to naturalism. So, if we hope to show that non-qualitative properties are consistent with naturalistic metaphysics, we must show that, when providing a metaphysics for our best scientific theories, we can retain individualistic facts and, in turn, non-qualitative properties. With this in mind, I'll now turn to several strategies for resisting Dasgupta's argument in the next section.

\section{§4. Qualitativism and De Re Laws}

What would it take to undermine Dasgupta's argument and, in doing so, defend the naturalistic acceptability of individuals and, in turn, non-qualitative properties? One option would be to show that, contrary to Dasgupta, individuals are, in fact, detectable. Pursuing this option requires us to tackle thorny questions about the nature and extent of detectability. According to Dasgupta, the relevant sense of "detectable" is singled out by direct appeal to scientific practice: whatever physicists mean when they claim that absolute velocity is "undetectable" is just what Dasgupta means when he claims individuals to be undetectable. Despite this, we still seem far from an especially clear account of detectability. This is due, in part, to the fact that detectability is a modal notion and subject to the familiar vicissitudes of context-sensitivity. Among other things, when we consider which entities are detectable, we are forced into taking a stand on which possibilities are "properly disregarded." Notice, for example, that in ordinary contexts, we can properly

disregard possible worlds in which God's voice repeatedly booms across the universe 
announcing that this or that haecceity is instantiated by a certain particle. So, even while there are some worlds where individuals could be detected, the intended sense of "detectability" requires us to ignore such far off worlds as irrelevant. Consider, however, Michael Tooley's peculiar case regarding Smith's garden:

Suppose, for example, the world were as follows. All the fruit in Smith's garden at any time are apples. When one attempts to take an orange into the garden, it turns into an elephant. Bananas so treated become apples as they cross the boundary, while pears are resisted by a force that cannot be overcome. Cherry trees planted in the garden bear apples, or they bear nothing at all. If all these things were true, there would be a very strong case for its being a law that all the fruit in Smith's garden are apples. And this case would be in no way undermined if it were found that no other gardens, however similar to Smith's in all other respects, exhibited behavior of the sort just described. (Tooley (1977: 687))

In a world with Smith's garden (or parallel phenomena regarding a specific spacetime point or a spooky fundamental particle), we would be faced with qualitative patterns in nature that demand explanation yet resist systematic explanation in purely qualitative terms..$^{22}$ Under such conditions, we would have grounds for positing de re laws-laws involving specific individuals like Smiththat require individuals and the non-qualitative properties that distinguish them. While the actual world does not seem to be governed by any de re laws, Tooley contends that such laws could have obtained. ${ }^{23}$ And, if they did, we would seem to have a means for detecting at least some individuals like Smith. ${ }^{24}$

Does the mere possibility of a case like Smith's garden show that Dasgupta is mistaken when he claims that individuals are undetectable? Probably not. Even if there is an intuitive difference between bizarre worlds where God is constantly yelling out the names of individuals and nomically interesting ones with Smith's garden, metaphysical possibilities of each sort can still be properly ignored when we assert that individuals are undetectable. Indeed, it seems that, for Dasgupta, we can properly disregard any nomologically impossible worlds. But, while cases like Smith's garden do not undermine Dasgupta's claim that individuals are undetectable, accommodating their metaphysical possibility can, as I'll now argue, generate a different problem for qualitativists.

If we take recent philosophical history as a guide, the orthodox response to a case like Smith's garden is to reject its metaphysical possibility out of hand. For some, this response is motivated by a conception of scientific laws on which generality is a necessary condition for lawhood. This follows in the tradition of Hempel and Oppenheim and is put succinctly by J.J.C. Smart (1963: 53): "laws are universal in that it is supposed that they apply everywhere in space and time, and they can be expressed in perfectly general terms without making use of proper

\footnotetext{
22 Dasgupta (2009: 43) notes that possibility of interpreting Aristotelian physics as committed to de re laws. ${ }^{23}$ As Tooley (1977: 687) puts it, "'I am inclined to think, then, that it is logically possible for there to be laws and nomological statements, in the strict sense, that involve ineliminable reference to specific individuals." ${ }^{24}$ Not every de re law ensures the detectability of individuals. Some laws might involve undetectable properties or relate networks of undetectable individuals. I set aside this complication here.
} 
names or of tacit reference to proper names." ${ }^{25}$ For others, the ban on de re laws flows, not from considerations regarding laws, but from the nature of metaphysical possibility and the extent of de re necessities. For instance, Kit Fine (2005: 243) rules out de re laws concerning specific individuals, saying: "Although I have emphasized the way in which natural necessities may outrun the metaphysical necessities, it seems to me that there is one respect in which this may not be true. For I am inclined to think that there are no distinctive de re natural necessities."

The prohibition against de re laws is understandable enough. After all, countenancing such possibilities yield several challenges for views about the nature of laws and metaphysical necessity. Among other consequences, de re laws wreak havoc on views that hold the relata of natural laws to be qualitative properties. But, if the case of Smith's garden (or one comparable to it) satisfies our general standards for conceivability arguments, we cannot justifiably reject its possibility solely to dodge theoretical complications for our preferred account of laws. And, when compared to conceivability arguments that enjoy currency in other metaphysical debates, there seems to be rather little to find suspicious in Smith's garden case. Perhaps more importantly, if we interpret Aristotelian physical theory as requiring de re laws regarding motion around the center of the cosmos, de re laws will have been front and center throughout a remarkably long stretch of Western physical theory. Coupled with the conceivability of Smith's garden, a defeasible presumption in favor of the mere possibility of a once-leading physical theory suggests we ought to believe that at least some de re laws are genuine metaphysical possibilities.

Let us assume, then, that de re laws are possible. It follows that there are possible worlds in which individuals exist. For those who claim that individualism is a necessary truth, de re laws are easy enough to accommodate. There are some worlds like the one with Smith's garden in which individuals are detectable, but, in other worlds individuals exist while remaining undetectable. For qualitativists, matters are importantly different. For, if de re laws are possible, qualitativism can be only contingently true. There are therefore individualistic possible worlds as well as qualitativist possible worlds. And, rather strikingly, some of these worlds will be qualitatively indistinguishable from one another despite differing at the level of fundamental metaphysical structure. For example, if qualitativism is actually true, there will be an individualist possible world with the very same qualitative character but in which individuals exist despite being undetectable.

A problem for contingentist qualitativism now arises. This problem results from admitting both individualist and qualitativist worlds and the extreme metaphysical variation this requires. For, if there are worlds of each kind, there seems to be no non-ad hoc reason to deny the possibility of "patchwork worlds," where individualistic facts are arbitrarily distributed amongst qualitative ones. ${ }^{26}$ Modest assumptions about the recombination of possibilities will, for example,

${ }^{25}$ Hempel and Oppenheim (1948: 157) describe this constraint as follows: "There can be little doubt, however, that there exists a large number of property and relation terms which would be rather generally recognized as purely qualitative in the sense here pointed out, and as permissible in the formulation of fundamental lawlike sentences; some examples have been given above, and the list could be readily enlarged. When we speak of purely qualitative predicates, we shall henceforth have in mind predicates of this kind."

${ }_{26}$ According to Dasgupta (2009: 55, 58), generalism is a holistic thesis regarding the entirety of reality. Such a view requires us to reject familiar combinatorial assumptions about modality-e.g., that we might 
entail that there are worlds where an individual occupies your qualitative role, but there is no individual that occupies the qualitative role of your next door neighbor or your mailbox. If we express fundamental facts via sentences like (World) or corresponding Ramsey sentences like $\left(\right.$ World $\left.^{*}\right)$, even quite weak combinatorial principles suggest that there are also possible worlds of the following sort:

$$
\text { (Patchwork) } \exists x(F x \& G x) \&(F a \& \sim G a) \& \sim(x=a)
$$

Contingentist qualitativists seem to be without good reason to deny the possibility of worlds with fundamental facts like (Patchwork), where an individual "occupies" one qualitative role but another qualitative role is simply left unoccupied. And, in such patchwork worlds, the nonqualitative properties that distinguish individuals seem all but arbitrarily sprinkled over the mosaic of qualitative properties.

There is little to like about a metaphysics that admits this degree of structural variation. Among other things, it generates an unwelcome species of metaphysical skepticism. In addition to epistemic concerns about how to discern whether the actual world is individualist or qualitativist, the contingent qualitativist faces an even more awkward question: how can we tell which of the qualitative roles in our world are actually occupied by individuals? For instance, does my dog, Edie, really exist or is there merely qualitative structure where I take her to be? Pressed to admit patchwork worlds that follow on the heels of de re laws, qualitativist metaphysics becomes increasingly unattractive. In contrast, if individualism is a necessary truth, the individualist is comfortably positioned. She can account for worlds with de re laws, although there is no analogous case that mandates qualitativism, since the structure of individualism is inherently richer than that of qualitativism. She can therefore concede that, in certain worlds, individualistic facts are unknowable, but she can also avoid the patchwork worlds that come with contingent qualitativism. More generally, since contingent qualitativism requires that the fundamental laws of metaphysics - those regarding individuals and properties - vary not just from world to world but even across times and regions within a single world, it turns out to be an unattractive, exceptionally disunified metaphysics. On balance, then, individualism offers a preferable treatment of metaphysical structure once we attend to the full range of laws that could have obtained.

There is, however, a $t u$ quoque still available to the qualitativist: Suppose that combinatorial reasoning does require the contingent qualitativist to accept patchwork worlds. Why think that the individualist is not similarly saddled with patchwork worlds? What licenses the individualist to claim that her view is a necessary truth and, in doing so, reject the possibility of qualitativist worlds? When pressed in this way, the individualist's options are somewhat limited. She can point out that qualitativism is an exceptionally heterodox metaphysical option, which likely requires an unfamiliar, ideologically bloated metaphysical structure that fares

recombine portions of the world to yield non-actual possibilities. Here, I assume a modest combinatorialism which requires patchwork worlds, but I leave aside scrutiny of the extent to which qualitativism requires a decidedly anti-Humean conception of modality that will eschew any degree of recombination. 
poorly with respect to theoretical virtues like conservativism. Moreover, if individualism is viewed as a necessary truth, we can provide a unified account of the metaphysical structure of worlds with and without de re laws. She can also note that every metaphysician is forced to draw a line between metaphysical possibilities and merely epistemic ones and that this line seems most naturally drawn at the level of fundamental metaphysical structure regarding ontological categories like individuals and properties. These are significant considerations, but the burden of proof is difficult to assess here. And, if individualists are forced to accept qualitativist worlds as well as the patchwork worlds that follow on their heels, the case for preferring individualism over qualitativism will collapse.

Notice, however, that even if (necessitarian) individualism allows for a unified, elegant metaphysical theory that readily accommodates de re laws, it is unclear that such considerations might compel naturalists. After all, the theoretical unification and elegance that individualism secures is concerned with a subject matter of little interest to scientific theory-namely, nonactual worlds with laws quite different from the actual one. It is therefore unlikely that naturalists will be moved by such theoretical benefits, since these considerations seem extra-scientific in character. Consider, for example, a physical parallel: suppose a physicist were to claim it as a key virtue of her theory of gravitation that it neatly accounts for what happens in merely possible worlds stuffed with alien ectoplasm. It's hard to imagine that physicists would recognize this as a genuine or substantial virtue. So, although worries about de re laws and contingent qualitativism might provide non-naturalists with reason to prefer individualism, our central ambition-of showing that naturalists ought to retain non-qualitative properties - is still unsatisfied, since naturalists are within their rights to ignore the above concerns regarding how best to accommodate exotic metaphysical possibilities.

\section{§5. Qualitativism and Haecceitism}

Arguments premised upon non-actual de re laws are unlikely to generate the naturalistic considerations needed to overturn Dasgupta's argument. Importantly, this isn't simply because such cases involve an appeal to modal notions. ${ }^{27}$ While there are naturalists who do harbor deep hostilities towards modality, such views are difficult to square with the pervasive appeal to modal notions across scientific theories, ranging from counterfactual reasoning in cognitive psychology, appeals to alternative world-states in equilibrium explanations, not to mention quotidian talk of dispositional properties like malleability. The real reason why considerations stemming from de re laws and cases like Smith's garden will leave naturalists unmoved is because such possibilities crucially depend upon controversial appeal to alternative laws of nature and, in doing so, assume that metaphysical possibility outstrips nomic possibility. In this section, we will consider two different lines of response to Dasgupta's argument, each of which seeks to establish the need to posit individuals in order to make sense of certain genuine nomological possibilities. In each case, the possibilities in question imply the reality of haecceitistic differences-i.e., exclusively non-qualitative differences between maximal

\footnotetext{
${ }^{27}$ For Quinean naturalists, modality is, by its very nature, disreputable and rightly evicted from scientific theories. On the motivation for and prospects of Quinean opposition to modality, see Burgess (1998).
} 
possibilities. ${ }^{28}$ So, if we are to accommodate such differences, we require individuals along with their respective non-qualitative properties.

Let's start with a case drawn from Melia (2003: 162). ${ }^{29}$ Consider a world containing only a qualitatively homogeneous circular plane and, at the center of this circular plane, a qualitatively homogeneous cylinder. At some time, the cylinder topples over and comes to rest. Intuitively, there are many directions in which the cylinder could have fallen. But since there are no qualitative differences between these possibilities, accounting for their distinctness requires metaphysical structure in addition to qualitative structure-namely, that there are individualist facts about the identity of specific regions of the homogeneous plane. Unless we are willing to abandon our initial modal judgment that there are distinct directional possibilities for the cylinder, the distinctness of these possibilities requires that there are maximal possibilities (or, alternatively, possible worlds) that differ only non-qualitatively-i.e., with respect to the identity of the regions on the plane that are occupied by the fallen cylinder. Individuals are therefore needed to account for modal phenomena - in this case, distinct directional possibilities.

The cylinder case poses a direct challenge for qualitativism. . If we assume that the accommodation of alternative physical possibilities governed by actual laws is a naturalistic requirement, then accounting for the differences betweem these directional possibilities could provide grounds for positing individuals. Unfortunately for the individualist, the qualitativist is well-positioned to resist this kind of haecceitistic argument. ${ }^{30}$ In particular, the qualitativist can plausibly claim that any argument of this sort yields, at best, a deadlock and, at worst, begs the question against qualitativism.

To see why, notice that the key premise in the cylinder-based argument for individualism is a modal one: that there are distinct directional possibilities for the physical system in question. . According to the qualitativist, the support for this modal premise issues, not from the genuinely naturalistic content of our best mechanical theory, but instead from extra-scientific modal intuitions about physical systems. The qualitativist will therefore deny this modal claim, insisting that there is no naturalistic pressure to accommodate our naïve modal intuitions regarding physical systems. We only need to make good on the possibilities that issue from our best physical theories and their most plausible interpretations. Since the qualitativist holds that the best interpretation of our physical theories eschews individuals, the deployment of these theories in any modal context cannot generate a new mandate in favor of positing individuals. And, for the qualitativist, this means we have no naturalistically credible reason to impute individuals into the cylinder world. According to the qualitativist, the principled, naturalistic stance to adopt is one that holds there to be a unique directional possibility for the cylinder.

${ }_{28}$ Complications abound here regarding options for interpreting or accommodating haecceitism. For metaphysical options regarding haecceitism, see Lewis (1986), Skow (2009), and Cowling (2015).

${ }^{29}$ Controversy abounds over the relationship between substantivalism about spacetime, haecceitism, and determinism-most notably, in the literature surrounding the Hole Argument. For discussion, see Brighouse (1997).

${ }^{30}$ A quick anecdote: Lynne had a knack for being helpful and self-deprecating within a single breath. I wrote my dissertation on haecceitism. At the outset of my dissertation defense, I was racing through a summary of some chapters. Lynne, visibly amused, stopped me mid-sentence: "You sound worse than a Southerner! You keep saying 'hex-itism' but you're missing half the darn syllables! It's 'heck-see-uh-tism.'” 
Although individualists will claim that this stance runs afoul of our familiar modal judgments, notice that qualitativist have a potent response. In the spirit of Dasgupta's argument, the qualitativist can argue that multiplying possibilities only serves to further illustrate the epistemic problems for individualism. Since we remain without any means for detecting individualistic facts, it is unclear how-even supposing we could somehow insert epistemic agents into a world while retaining the relevant symmetries - we might go about distinguishing among these putatively distinct directional possibilities. This point is an important one: if we posit individuals solely to explain the numerical distinctness of possibilities, this will do nothing to undermine Dasgupta's claim that we are irremediably ignorant of individualistic facts. Sustaining haecceitistic differences corresponding to directional possibilities will therefore be a hollow victory, since we are still left without any inkling of how we might discern among these newly multiplied possibilities. It looks, therefore as though the cylinder case, like Smith's garden case, does not provide compelling naturalistic grounds for positing non-qualitative properties.

Let's step back for a moment. In presenting his case for the undetectability of individuals, Dasgupta takes up the question of whether we have the epistemic resources required to discern individuals and individualistic facts. In doing so, Dasgupta distinguishes two different senses in which we might know or detect individuals: the ordinary sense that might arise in, say, discerning which chair is which from among a dining set and the narrow sense relevant here, which concerns our (in)ability to "empirically detect which primitive individual [the chair] is." (45) As we've seen, Dasgupta claims that the individualist is committed to a view on which "there is a further fact of the matter concerning [the chair's] identity"(45) but that, given what we know about physical theory, detecting such a fact is impossible. Dasgupta's contention is that scientific inquiry is insensitive to de re or individualistic matters and, as a consequence, justified belief in individualistic facts is endemically unwarranted. This seems plausible enough once we note that our capacity to detect and discern individuals can seem wholly dependent upon the qualitative features of things-e.g., where and when they are and what they are like. So, given naturalistic principles of theory choice that caution against admitting undetectable posits, the qualitativist concludes that we ought to reject individuals and individualistic facts.

I am sympathetic with some of Dasgupta's assessment: there is no reason to believe that we have the epistemic means to detect individualistic rather than qualitative facts regarding most individuals. We cannot see haecceities. Nor can we discriminate between duplicates and doppelgangers through some purely non-qualitative sense. For this reason, our epistemic access to individualistic facts is far more tenuous than is typically assumed. But, while Dasgupta is correct that we have no general de re sense for detecting individualistic facts, he omits the distinctive kind of de se access we have to a narrow class of individualistic facts regarding our own identity. We can, for example, form justified beliefs regarding certain individualistic facts in virtue of being perspectival beings. Among other things, we can distinguish between whether a certain experience has befallen us rather than another individual and, as a consequence, we have reason to believe that some individualistic facts are detectable albeit "from the inside." Indeed, as I'll now argue, the fact that we have first-person perspectives is precisely what puts us in an epistemic position to detect certain individualistic facts. While this shows that some individualistic facts are detectable, it also confirms a methodological moral stressed by Baker: first-person perspectives are indispensable when doing metaphysics. Even if they are not 
ontologically basic as Baker claims, perspectives afford us distinctive access to certain features of reality that ought to inform our fundamental metaphysics.

To see why de se matters warrant special attention here, recall that, as perspectival beings, we are in a position to consider possibilities either third-personally ("from the outside") or firstpersonally ("from the inside"). ${ }^{31}$ And, as Adams (1979) notes, when we evaluate certain conceivability arguments for haecceitism, the reality of certain haecceitistic differences becomes "vividly clear" only after we consider the relevant possibilities "from the point of view of a person." Although Adams' primary concern is with making the case for haecceitism, his insight points us toward a general conclusion: certain aspects of metaphysical structure are obscured if considered only from the outside, but become manifest when we experience or imagine from the inside. Consider the following case which Adams (1979: 22) offers in defense of haecceitistic differences between possible worlds:

Consider, again, a possible world $w_{1}$, in which there are two qualitatively indiscernible globes; call them Castor and Pollux. Being indiscernible, they have of course the same duration; in $w_{1}$ both of them have always existed and always will exist. But it seems perfectly possible, logically and metaphysically, that either or both of them cease to exist. Let $w_{2}$, then, be a possible world just like $w_{1}$ up to a certain time $t$ at which in $w_{2}$ Castor ceases to exist while Pollux goes on forever; and let $w_{3}$ be a possible world just like $w_{2}$ except that in $w_{3}$ it is Pollux that ceases to exist at $t$ while Castor goes on forever. That the difference between $w_{2}$ and $w_{3}$ is real, and could be important, becomes vividly clear if we consider that, from the point of view of a person living on Castor before $t$ in $w_{1}$ and having (of course) an indiscernible twin on Pollux, it can be seen as the difference between being annihilated and somebody else being annihilated instead. But there is no qualitative difference between $w_{2}$ and $w_{3} .{ }^{32}$

In presenting this argument for haecceitism, Adams notes, first, that we can imagine two qualitatively indiscernible possibilities for a symmetric universe with two globes, Castor and Pollux. In one world, Castor explodes and, in a second world, Pollux is destroyed. And, although the distinctness of these possibilities might seem tenuous when we imagine them from the outside, the difference between them proves manifest once we imagine them from the inside by imaginatively placing ourselves on, say, Castor. For, once we suppose ourselves to occupy a specific globe, the difference between these possibilities is about as profound as it gets, since it is marked by whether or not we survive. It is, however, a purely individualistic difference concerning whether it is me or, instead, my qualitatively indiscernible twin that is destroyed. Importantly, this difference is obscured if we neglect to imagine these scenarios from the vantage point of a perspectival being that occupies a specific location within the relevant possible worlds.

If Adams is correct, there are possible worlds divided by individualistic facts. Moreover, the difference between these worlds can be detected by those in them and, in turn, imagined from

31 On imagination and the inside/outside distinction, see Lewis (1979).

32 Cf. Melia (1999: 650): “we could imagine a collection of bald philosophers, sitting in a circle. It is a law that one of them will grow a single hair. But, by the symmetry of the situation, any of the philosophers could be the lucky one." Now consider whether an individualistic difference would be detected if you, while staring straight ahead, were to see a hair sprout from a philosopher's head. 
the inside once we note that these worlds include perspectival beings like us. Since we can tell whether we are in a world in which it is us rather than our qualitative doppelganger that is destroyed, Dasgupta is only partly correct about the epistemology of individualistic facts. Although no inspection of reality "from the outside" - no matter how exhaustive-would seem to provide justified beliefs in specific individualistic facts, individualistic differences between worlds are nevertheless detectable for bearers of perspectives. We should therefore reject Dasgupta's claim that individualistic facts are always beyond our epistemic access. We can, under the right conditions, form justified beliefs about reality that concern our continued existence and such facts are not qualitative. They are, instead, purely individualistic yet capable of grounding haecceitistic differences between possible worlds.

Notice that, Adams' "global destruction" case provides something that the cylinder case cannot: an account of how we could have epistemic access to the individualistic facts that ground the haecceitistic differences between possibilities. While we are without any account of how we might detect the difference between distinct directional possibilities regarding the cylinder in its symmetric worlds, the difference between the global destruction possibilities is manifestly accessible to those with perspectives. And, although Adams' case assumes the possibility of symmetric worlds, it does not require any exotic suppositions about non-actual laws of nature. Naturalists cannot therefore dismiss Adams' argument as engaging in unduly exotic metaphysics as they might hope to dismiss Tooley's case regarding Smith's garden.

At this point, some naturalists might be tempted to suggest that the individualistic facts regarding the survival of specific individuals are not "properly" detectable. They are not, for example, amenable to empirical study from an arbitrary third-personal position. And if we imagine worlds that differ in this way only "from the outside," there does seem to be minimal temptation to distinguish between them. Naturalism that denies the reality of facts accessible only from specific perspectives does not, however, have much to recommend in its favor. While firstpersonal phenomena-most obviously, psychological phenomena-must be carefully handled in scientific inquiry, it is difficult to believe that naturalists ought to accept an epistemology according to which we are unable to determine whether or not we continue to exist-i.e., detect our own existence-on the basis of our perspectival experience. If naturalism requires that all facts are epistemically accessible from the outside, there is little plausibility in the epistemology of naturalistic metaphysics and, for such versions of naturalism, Baker's withering skepticism seems the apt treatment.

Adams' case provides compelling evidence for haecceitistic differences between possibilities. Such differences require non-qualitative properties. Better still, such a case comes with a plausible account of how we might detect the individualistic facts that divide these possibilities. Is this - finally-enough to show that naturalists ought to accept the actual existence of non-qualitative properties? Unfortunately, not quite. Even if Adams' case provides reason to believe that there are some possible worlds where individualism is true (or, indeed, pairs of such worlds that differ haecceitistically), it is still open to the naturalist to insist that, here, at the actual world, qualitativism is still true. And, as we saw earlier, it is far from clear how worries about the contingency of qualitativism might be used to compel naturalists to opt for the necessary truth of individualism. So, although Adams' case might provide reason for non-naturalists to abandon 
qualitativism, naturalists are still under little, if any, pressure to endorse individualism and admit non-qualitative properties.

There is, however, one remaining line of argument to be considered, which I set out in the next section. And, unlike those we've considered so far, this argument does seem to show that naturalists are obliged to posit individuals and non-qualitative properties.

\section{§6. Naturalism, Nominalism, and Qualitativism}

Dasgupta's case against individualism proceeds from a suggestive analogy between individuals and absolute velocity. In this section I turn to a different analogy - one between individuals and mathematical entities - in an effort to show that naturalists ought to retain a commitment to individuals and therefore non-qualitative properties. I'll begin by setting out some key features of the debate over mathematical nominalism when undertaken in a naturalistic context. ${ }^{33}$

Mathematical nominalists deny the existence of abstract mathematical entities like numbers, sets, and functions. Mathematical platonists affirm the existence of such entities. For naturalists, this ontological disagreement hinges upon a central question: does a naturalistic commitment to our best scientific theories require belief in the existence of mathematical entities? For proponents of the Indispensability Argument, naturalistic commitment to our best scientific theories is sufficiently robust that, given our literal acceptance of scientific theories and their indispensable quantification over mathematical entities, platonism is a mandatory ontological consequence. Rejecting the existence of mathematical entities would entail that we reject the mathematical claims of our best scientific theories. But, since our belief in these mathematical claims is no less warranted than belief in parallel claims regarding physical entities, any reason to reject mathematical entities runs contrary to naturalist's fealty to the verdicts of our best scientific theories. Let's call this attitude towards mathematical entities naturalistic platonism.

The most influential nominalist challenge to naturalistic platonism owes to Hartry Field. Field (1980) sets out a strategy for "nominalizing" Newtonian gravitational theory in an effort to show that mathematical entities are, in fact, eliminable from physical theory. Roughly put, Field's program replaces quantification over mathematical entities with a range of ontological and ideological resources including substantivalism about spacetime, classical extensional mereology, and a variety of ideological primitives. Field subsequently argues that, since nominalists can formulate a nominalized "replacement" of our best physical theories, naturalists can coherently disavow the existence of mathematical entities by replacing mathematical claims with a variety of non-mathematical ones. Belief in the non-mathematical portions of our best scientific theories can therefore be retained even while nominalists of this sort hold that mathematical claims, including arithmetic, are, strictly speaking, false.

This all too brief summary belies the complexity of Field's nominalization strategy. Among other issues, Field's program raises the difficult question of which resources are nominalistically acceptable and whether this general strategy might be extended to all of our best physical theories. But, if we assume Field's program is not confounded by these or other

\footnotetext{
${ }^{33}$ For example, we can set aside Eleatic arguments based upon a priori principles that link existence with causation. For a survey of arguments for and against nominalism, see Cowling (2017).
} 
complications, the main question is whether platonism remains a mandatory commitment for naturalists or if naturalists are within their rights to disavow mathematical entities.

Responses to Field's nominalism are numerous, but one is of particular significance for our purposes. It takes the form of a pointed challenge set out in Burgess (1983) and Burgess and Rosen (1997). Suppose that Field's reformulation of physical theories is a sufficiently rich and inferentially suitable replacement for platonistic physical theories formulated with quantification over numbers. ${ }^{34}$ Even granting this much, would the resulting replacement really be among our best scientific theories? Notice that these nominalist replacement theories are almost certainly less familiar, more cumbersome to formulate, and, perhaps most importantly, motivated, not by considerations internal to physics, but by extra-scientific reservations regarding abstract entities. ${ }^{35}$ Why, then, should scientists ever prefer-much less treat as equal-these nominalized replacement theories when extant platonist ones are available? How could any such replacement theory be of genuine appeal to a dyed-in-the-wool naturalist?

Burgess and Rosen (1997: 206) present this challenge to the nominalist in especially stark terms by asking how we might expect physicists to assess the result of Field's labors: "For ultimately the judgement on the scientific merits of a theory must be made by the scientific community: the true test would be to send in the nominalistic reconstruction to a mathematics or physics journal, and see whether it is published, and if so how it is received...." Burgess and Rosen then point out that "This, however, is a test to which, so far as we know, none of the nominalists whose work we have surveyed has subjected himself. (At any rate, no papers on nominalistic physics have appeared in the journal mentioned.) And it is easy to understand why, since it is easy to imagine what the reaction to the submission of such a paper to such a journal would be."

The challenge that Burgess and Rosen pose here is a grave one for naturalists suspicious of mathematical entities as well as any other naturalists that harbor plans to defend revisionary metaphysical theses. As they put it,

[I]f the nominalistic reformer is to claim to be an adherent of naturalization or naturalism in epistemology, the 'naturalism' in question must be of a restricted variety, making invidious distinctions, marginalizing some sciences (the mathematical) and privileging others (the empirical). And it must also be of a selective variety, again making invidious

\footnotetext{
${ }^{34}$ Burgess and Rosen present this challenge as a dilemma, leaving open that naturalist nominalists might pursue an "hermeneutic" approach which claims that we have been "nominalists all along." Roughly speaking, hermeneutic views hold that the correct view of mathematical discourse is one on which it leaves us uncommitted to the existence of mathematical entities (e.g., by virtue of being implicitly figurative). While I leave the individual analogue-hermeneutic qualitativism-undiscussed here, I take it to be incompatible with naturalism and notably different from the view Dasgupta defends. I therefore set aside hermeneutic options.

${ }^{35}$ The grounds Field (1980) presents for pursuing nominalism are of uncertain naturalistic standing. While he briefly remarks upon the epistemic challenges that arise for views on which mathematicalia are noncausal entities outside of space and time, he also cites two benefits of nominalist revisionary physical theories: a reduction in the arbitrariness that comes with the use of mathematical entities (e.g., as coordinate systems) and an increase in the extent to which physical explanations are "intrinsic," invoking only causally relevant physical entities but not acausal mathematical ones.
} 
distinctions, and down playing the importance of some norms (familiarity and perspicuity and fruitfulness) while playing up that of others (economy or parsimony, specifically or especially of abstract ontology)... What we want to add is that a thoroughgoing naturalist would take the fact that abstracta are customary and convenient for the mathematical (as well as other) sciences to be sufficient to warrant acquiescing in their existence. (211)

If Burgess and Rosen are correct, naturalism cannot abide revisionary metaphysics that rides roughshod over the current standards and practices of scientists. And, since reference to and quantification over mathematical entities is pervasive and undertaken without reservation in science, nominalism is incompatible with naturalism, understood as the view that our best scientific theories are the ultimate arbiter of ontology.

Burgess and Rosen's challenge for nominalists suggests a general moral: naturalist metaphysics prohibits ontological revisionism-namely, disagreement with the apparent commitments of extant scientific standards and practices. In effect, nominalists and other ontological revisionists cannot really be naturalists. What, then, should we make of qualitativists who seek to dispense with individuals?

If the qualitativist is to avoid a challenge that runs parallel to the one Burgess and Rosen pose for the nominalist, she must show one of two things. First, that qualitativism isn't really revisionary; that it doesn't conflict with the extant standards and practices of scientists, despite the fact that scientists certainly seem to think, speak, and reason as though there are individuals. Alternatively, she might hope to show that extant scientific standards and practices really do warrant revision on genuinely scientific grounds. This second option would amount to a demonstration that it is Dasgupta's argument and resulting qualitativism that is the proper outcome of correctly applying scientific standards while, in contrast, the flat-footed retention of individuals, merely seems to be a naturalistic commitment. ${ }^{36}$

To see that this first option is of little promise notice that appeal to individuals is endemic to the sciences. Not only do we find rampant talk of individuals across the sciences, we even find pervasive appeal to specific individuals like Pangea and Chicxulub in historical sciences. There is, then, no apparent reason to think that the use of singular terms is, in general, a contentious matter for scientists. To be sure, there are myriad scientific debates about whether there are certain entities and whether we our theories ought to shuffle certain posits like spacetime across ontological categories, but none of this is premised upon naturalistic reservations about the coherence of individuals qua individuals. So, absent any serious evidence of metaphysical reservations about individuals, there is considerable pressure for naturalism to mandate the retention of individuals in general.

Any hope for a naturalistically credible version of generalism therefore hangs on whether the second option described above is a viable one. This, in turn, raises the question of whether the key principle of theory choice to which Dasgupta appeals - namely, the defeasible prohibition against redundant and undetectable entities - is sufficient grounds to override all of the virtues

\footnotetext{
${ }^{36}$ As noted earlier, the qualitativist could depart from Dasgupta's generalism by retaining quantificational structure while rejecting individuals. Such a view avoids some challenges for generalism, but still runs afoul of the pervasive occurrence of names throughout scientific practice. Given their similar pitfalls, I take it that qualitativism and generalism to be more or less interchangeable here. .
} 
that attend individuals. And, here, it seems enough to point to Burgess and Rosen's parallel description of the state of the debate over nominalism, since the qualitativist, much like the nominalist, seems guilty of "downplaying the importance of some norms (familiarity and perspicuity and fruitfulness) while playing up that of others (economy or parsimony...)"37 Moreover, just as Burgess and Rosen point out that the proof of nominalism's merits is in the pudding of its reception, we ought therefore to ask the parallel question regarding qualitativism: what would a physics journal make of a paper presented in the language of functorese, which is required by the qualitativist seeking to dispense with individuals? ${ }^{38}$ The metaphysical virtues of such an approach simply would not translate into recognizable scientific merits and puzzlement by those grappling with the qualitativist replacement theory seems exceptionally likely. ${ }^{39} \mathrm{We}$ need not deny that Dasgupta's argument points to a genuinely naturalistic consideration, but it is only a single consideration among many. And, when set against the larger matrix of disciplinary standards, it is unable to bear the weight necessary for undermining the individualistic metaphysics that naturalism requires.

It looks, then, like naturalists are obliged to retain a metaphysics of individuals in much the same manner that they are obliged to sustain a metaphysics of mathematical entities. ${ }^{40}$ And, with individuals comes a commitment to non-qualitative properties. The upshot, for those of us interested in finding a place for the first-person perspective, is that there is no good reason to believe that haecceities and other non-qualitative properties are prohibited by naturalism. On the contrary, for what Burgess and Rosen call "thoroughgoing naturalists," they are mandatory. ${ }^{41}$

37 One might seek to extend Dasgupta's argument by claiming that, since mathematical entities are undetectable and physically redundant, they should also be rejected by naturalists. Such an argument turns upon the proper application of principles regarding theory choice across physics and mathematics. Of course, if numbers are individuals and naturalism requires platonism, we have a direct route for challenging qualitativism though only in the abstract realm.

${ }_{38}$ On the details of functorese, see Quine (1976), Turner (2011) and Dasgupta (2009).

${ }^{39}$ Note that my claim isn't that naturalists might not have some specific use for functorese or that naturalism could, given future developments, mandate qualitativism either as a general thesis or one regarding a specific target phenomenon. My point here is that qualitativism, with its full-scale rejection of individualism, is presently without sufficient motivation via naturalistic grounds.

40 Recall that Dasgupta's argument focuses on what he calls "primitive individuals." A potential qualitativist response might therefore seek to limit qualitativism to fundamental reality and accept a metaphysics of non-fundamental individuals. Roughly put, such a view would deny that there are fundamental particles or regions, but affirm the existence of tables and tadpoles. While this option is certainly open to non-naturalists, the distinction between the fundamental and non-fundamental is of highly uncertain naturalistic credibility. Even if we might permit it within our metaphysical theories, it is unclear how such a distinction might vindicate qualitativism from the argument offered here, especially given that "individualizing" is pervasive across the sciences, extending from talk of particles and spacetime regions to biological individuals and geological structures.

${ }^{41}$ Naturalists might object to this way of putting this conclusion. For, if we're naturalists, why concern ourselves with the decidedly metaphysical distinction between the qualitative and the non-qualitative? Can't we simply dismiss this distinction as "pure metaphysics"? Of course. The naturalist is within her rights to jettison the qualitative/non-qualitative distinction provided it doesn't show up in scientific practice. But, for naturalists, nothing much will hang on this result, since we've only used the distinction 


\section{§7. Conclusion}

I've argued that non-qualitative properties, like phenomenal and semantic properties, are part and parcel of the first-person perspective. Our interest in reconciling first-person perspectives with a naturalistic metaphysics therefore requires assessing whether naturalists can accept nonqualitative properties like haecceities into their ontology. To this end, we examined Dasgupta's argument against the existence of individuals. Given the relationship between individuals and non-qualitative properties, this argument, which holds individuals to be undetectable and physically redundant, seems to be the strongest naturalistic case against non-qualitative properties.

In examining potential responses to Dasgupta's argument, we considered arguments for the existence of individuals based upon the possibility of de re laws as well as various sorts of haecceitistic differences. And, while some of these arguments might sway non-naturalists who seek to avoid contingent qualitativism, certain kinds of haecceitistic differences, when imagined "from the inside," do seem to show that individualistic facts turn out to be detectable. Whether arguments that draw upon such cases will convince ardent naturalists to reject qualitativism is unclear, but, as I've argued, there is another more direct naturalistic case for individuals and, in turn, non-qualitative properties.

Drawing upon an analogy between debates over individuals and mathematical entities, I argued that the incompatibility of mathematical nominalism with naturalism parallels the tension between qualitativism and naturalism. So, although some principles regarding theory choice do bear against a metaphysics of non-qualitative properties, the cumulative naturalistic assessment of individualism and qualitativism suggests that naturalists ought to retain a metaphysics of individuals and, in turn, non-qualitative properties. So, if naturalism is incompatible with firstperson perspectives, we can conclude that it is not in virtue of a naturalistic hostility to the nonqualitative. If naturalism and the first-person perspective are opposed in the way Baker claims, it will be on account of some other kind of properties bound up with first-person perspectives. ${ }^{42}$

\section{§8. Works Cited}

here as a means to distinguish various properties like being Lynne Baker. If a naturalist rejects the qualitative/non-qualitative distinction, the present arguments can readily be recast as showing that naturalism is not at odds with properties like being Lynne Baker regardless of how one might categorize such properties.

${ }^{42}$ I am deeply grateful to Luis and Kevin for the opportunity to contribute to this volume. Toward the end of my time at UMass, Lynne was kind enough to let me sit in on her senior seminar on persons, right around the time she was writing Naturalism and the First-Person Perspective. It was a delight to watch her engage students with any number of foundational questions about personal ontology and identity. For me, it was an invaluable chance to appreciate her way of doing philosophy. In particular, I learned a lot about how "far out" metaphysics regarding haecceitism might bear upon the metaphysics of who and what we are. The present paper is an effort to work through a topic that regularly occupied our discussions-namely, how persons, perspectives, and haecceities were connected. Writing it was a powerful reminder of what it meant to do philosophy with her, how much she contributed to contemporary metaphysics, and how deeply her unique perspective is missed. 
Adams, R.M. 1979. “Primitive Thisness and Primitive Identity.” Journal of Philosophy 76: 5-26.

Baker, Lynne Rudder. 2013. Naturalism and the First-Person Perspective. Oxford: Oxford University Press.

Black, Max. 1952. “The Identity of Indiscernibles.” Mind 61: 153-164.

Brighouse, Carolyn. 1997. "Determinism and Modality." British Journal for the Philosophy of Science 48(4): 465-481.

Burgess, J. 1983. “Why I am not a nominalist.” Notre Dame Journal of Formal Logic, 24: 93-105.

--. 1998 "Quinus ab omni naevo vindicatus." in Kazmi, Ali (ed.) Meaning and Reference. Calgary: University of Calgary Press: 25-66.

Burgess, J. and Rosen, G. (1997) A Subject with No Object. Oxford: Oxford University Press.

Chalmers, David. 1996. The Conscious Mind. Oxford: Oxford University Press.

Cowling, Sam. 2015. “Non-Qualitative Properties.” Erkenntnis 80: 275-301.

--. 2017. Abstract Entities. London: Routledge.

--. (forthcoming) "Necessities beyond Necessity"

Dasgupta, Shamik. 2009. "Individuals: An Essay in Revisionary Metaphysics." Philosophical Studies 145: 35-67.

--. 2017. "Can We Do Without Fundamental Individuals? Yes." in Elizabeth Barnes (ed.) Current Controversies in Metaphysics. London: Routledge: 7-23.

Field, H. (1980) Science without Numbers. Princeton: Princeton University Press.

Fine, Kit. 2005. “The Varieties of Necessity,” in Tense and Reality. Oxford: Oxford University Press.

Friedman, Michael. 1983. Foundations of Spacetime Theories. Princeton: Princeton University Press.

Hempel, Carl, and Oppenheim, Paul. 1948. "Studies in the Logic of Explanation." Philosophy of Science 15(2): 135-175.

Jackson, Frank. 1998. From Metaphysics to Ethics. Oxford: Oxford University Press.

Ladyman, James, and Ross, Don with Spurrett, David, Collier, John. 2007. Every Thing Must Go. Oxford: Oxford University Press. 
Lewis, David. 1979. “Attitudes De Dicto and De Se.” Philosophical Review 88: 513-543.

Lewis, David. 1986. On the Plurality of Worlds. Oxford: Blackwell.

Melia, Joseph. 1999. "Holes, Haecceitism, and Conceptions of Determinism." British Journal for the Philosophy of Science 50: 639-664.

--. 2003. Modality. Montreal: McGill-Queen's.

Price, Huw. 2011. Naturalism without Mirrors. Oxford: Oxford University Press.

Simmons, Byron. (forthcoming) "Fundamental Non-Qualitative Properties" Synthese.

Skow, Bradford. 2009. "Haecceitism, Anti-Haecceitism, and Possible Worlds." Philosophical Quarterly 58: 98-107.

Smart, J.J.C. 1963. Philosophy and Scientific Realism. New York: Routledge.

Turner, Jason. 2011. “Ontological nihilism." Oxford Studies in Metaphysics, 8: 3-54.

--. 2017. “Can We Do Without Fundamental Individuals? No." in Elizabeth Barnes (ed.) Current Controversies in Metaphysics. London: Routledge: 24-42. 